\title{
This Special Issue Section of Silicon Showcases the Work of some of the Presenters at the American Chemical Society POLY Division Symposium 'Silicon-Based Hybrid Materials: Today, Tomorrow and the Future' that Took Place at the First Ever Virtual ACS National Meeting in August 2020
}

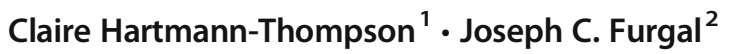 \\ Published online: 5 March 2021 \\ (C) Springer Nature B.V. 2021
}

The 'General Electric silicon alphabet' (M, D, T, Q) of silicon structures takes us from the organic to the inorganic realm by way of sol gel chemistry, and these molecular scale tools and structures enable a range of 'smart' macroscopic properties that can in turn enable us to build systems that solve realworld problems, and that address the mega-trends and challenges facing the world, all while limiting the use of petroleum feedstocks. Silicon chemistry, with its research taking off as a substitute for natural rubber during World War II, has always been notable for operating across the conventional boundaries between different fields of chemistry, and many rich examples of this truly 'hybrid' work were on display in the symposium.

Notably, novel ways of manipulating the making and breaking of bonds between silicon and other elements (particularly carbon and oxygen) via catalysis, photochemistry, chemoenzymatics and pyrolysis, are being further developed and new methodologies discovered. New metal catalysis chemistry is emerging by expanding the $\mathrm{Si}-\mathrm{C}$ bond-building toolbox beyond platinum-catalyzed hydrosilylation, and introducing catalysts based on earth-abundant metals such as iron, nickel, cobalt and manganese. New sol gel routes are being discovered that avoid the formation of problematic hydrolysis and condensation side products. Photochemistry is being leveraged for triggered 'on-demand' formation of Si-O bonds. For large surface area micro- or mesoporous structures,

Joseph C. Furgal

furgalj@bgsu.edu

Claire Hartmann-Thompson

chartmann-thompson@mmm.com

3M Corporation, St. Paul, MN, USA

2 Bowling Green State University, Bowling Green, OH, USA photo-responsive components can drive bond formation that enables light-driven pore dimension changes. These 'smart sponge' systems are capable of controlled uptake and release of species such as $\mathrm{CO}_{2}$, oils, organics and fluorochemicals, and have been demonstrated to function reversibly and 'return to baseline' through many cycles, opening many possibilities in environmental remediation and carbon capture. They may also be applied as photo-activable pumps, artificial muscles and drug delivery tools. Silicon-sulfur analogs of these porous networks (built from Si-S bonds) are being developed as gel polymer electrolytes for lithium sulfur batteries, taking advantage of low flammability and high ion mobility in sulfur systems.

Silicones share many useful properties with fluorochemicals, including high thermal stability, chemical resistance and low surface energy properties, and as fluorochemicals become more heavily regulated, there is a need to identify alternatives. Siloxanes effectively come from sand (via silicon metal and halo-silanes, but with new greener catalytic techniques being developed) and have the potential to degrade/oxidize back to sand. In the past, controlled Si-O bond breakdown has been an obstacle to silicone recycling, but new photochemical, metal catalytic, and/or enzymatic approaches are changing this, and are part of the transition away from single use plastics and the unintended formation of micro- and nano-plastic pollutants. Methods are also being developed to recycle silicones directly through self-healing mechanisms to prolong their useful life.

Silicones (denoted by the single letter D) are now more than half a century old, but exciting new variants are being developed. Slide-ring and concatenated ring architectures are being built with 'mobile crosslinks' that enable extreme elongations up to $5000 \%$ and transcend the conventional rubber entanglement plateau. Silicones are being functionalized with DNA bases that enable new modes of reversible elastomer 
cure and self-healing. Silicones functionalized with mechanochemical links that change property (such as color) in response to mechanical stimuli are finding myriad uses as stress sensors and actuators. Tough elastomeric variants of traditionally brittle polyionic liquid (PIL) electrolytes for stretchable batteries are being designed by introducing elastomeric silicone content. Methods of introducing magnetic functionality into 3D-printable silicones via smart encapsulation are being developed for soft robotics- overcoming the problem of high magnetic additive loadings being detrimental to mechanical properties.

Although our alphabet of M, D, T, Q building blocks can be combined to generate a near infinite range of structures (MQ resins, MT resins and silsesquioxanes, ladders and lanterns, bats and butterflies, polyhedra and Janus cubes, plus combinations with most of the minerals in the Earth's crust), much structure-property elucidation remains a work in progress. The structures of MQ resins are not fully understood despite their use in almost all commercial silicone adhesive products. The photochemical and photophysical adsorption, emission, and charge transfer processes associated with chromophore functionalized polyhedral silicon structures are still topics of on-going study. Likewise much structure-propertyprocess work continues in the area of silicon carbide ceramics and their polymeric carbosilane precursors, enabling the design of aerospace composites that can withstand increasingly hostile thermal shock, high temperature and oxidizing conditions.
There are also many outstanding challenges in surface chemistry to be addressed by silicones- taking new approaches based on interfacial slippage between layers and/or physical deformation- in both existing applications where the pool of acceptable fluoromaterials is diminishing, and in emerging applications such as anti-icing for wind turbines, and anti-biofouling in shipping and clean water infrastructure. Owing to their biocompatibility and low toxicity, silicones also remain the material of choice for wearable and medical use. Silicon-based oxygen-permeable hydrogels are continuing to dominate the contact lens market, and the area is expanding to include smart contact lenses with capabilities beyond vision correction, such as bio-responsive polymeric surfaces for anti-biofouling, light filters for light management applications, drug delivery, and monitoring of ocular physiology.

Silicon science will continue being a juggernaut in the development of high-performance materials that will have a substantial impact on humanity going forward. We have only demonstrated a few of the biggest and most influential areas being researched at this time, but the future of silicon-based materials in our world and beyond will play an ever-increasing role as we aim to curb the use of oil-based polymers and push the limits of civilization.

Publisher's Note Springer Nature remains neutral with regard to jurisdictional claims in published maps and institutional affiliations. 